\title{
Full counting statistics of renormalized dynamics in open quantum transport system
}

\author{
JunYan Luo, , f $\mathrm{Yu}$ Shen, ${ }^{1}$ Xiao-Ling He, ${ }^{1}$ Xin-Qi Li, ${ }^{2,}, 3$ and YiJing Yan $^{2}$ \\ ${ }^{1}$ School of Science, Zhejiang University of Science and Technology, Hangzhou 310023, China \\ ${ }^{2}$ Department of Chemistry, Hong Kong University of Science and Technology, Kowloon, Hong Kong SAR, China \\ ${ }^{3}$ State Key Laboratory for Superlattices and Microstructures, Institute of Semiconductors, \\ Chinese Academy of Sciences, P.O. Box 912, Beijing 100083, China \\ ${ }^{4}$ Department of Physics, Beijing Normal University, Beijing 100875, China
}

(Dated: October 22, 2018)

\begin{abstract}
The internal dynamics of a double quantum dot system is renormalized due to coupling respectively with transport electrodes and a dissipative heat bath. Their essential differences are identified unambiguously in the context of full counting statistics. The electrode coupling caused level detuning renormalization gives rise to a fast-to-slow transport mechanism, which is not resolved at all in the average current, but revealed uniquely by pronounced super-Poissonian shot noise and skewness. The heat bath coupling introduces an interdot coupling renormalization, which results in asymmetric Fano factor and an intriguing change of line shape in the skewness.
\end{abstract}

PACS numbers: 73.63.-b, 72.70.+m, 73.63.Kv, 73.23.Hk

\section{INTRODUCTION}

Electronic dynamics of an open quantum system has vital roles to play in the development of nanoelectronics [1 3]. Tremendous effort has been invested to characterize and control coherent dynamics in a variety of nanoscale transport systems [4 [9]. A lot of these studies are devoted to the energy relaxation and decoherence resulted from the coupling with a noisy environment [10-12]. Yet, another important consequence of systemenvironment coupling, i.e., internal energy renormalization, is attracting rising attention recently, owing to its essential influence on the internal dynamics of various nanoscale systems, such as spin valves 13 16, quantum dot (QD) Aharonov-Bohm interferometers [17, 18], double quantum dots [19, 20], and the quantum state under measurement [21, 22].

Full counting statistics (FCS), which characterizes the correlations between charge transport events of all orders [23, 24], serves as a 'spectroscopic' tool to reveal various internal mechanisms. In particular, owing to the development of highly sensitive on-chip detection of individualelectron tunneling technique, all statistical cumulants of the number of transferred particles can now be extracted experimentally. Electron transfer statistics in various nanoscale systems, such as QD [25 29], gold atoms [30], and molecules [31, 32], has been investigated and utilized to characterize the electronic dynamics. This offers new opportunities to study many-body effects such as the Coulomb blockade and Kondo dynamics with essential implications to nanoelectronics.

The main purpose of the present work is to relate the higher cumulants to the renormalized dynamical properties that cannot be accessed by the average current measurements. To have a specific example, we will inves-

*Electronic address: jyluo@zust.edu.cn tigate the energy renormalizations in electron transport through a double quantum dot system [33], where QD1 is directly tunnel-coupled to the electrodes while QD2 is side connected to QD1, as schematically shown in Fig.1. The double dots are further coupled to an inevitable dissipative phonon environment (not shown explicitly). The system is of particular interest, as it is in such a configuration that maximizes locality versus nonlocality contrast, and can be mapped onto nanostrucures in experiments [34, 35].

In context of FCS, we are capable of identifying the essential difference between the internal energy renormalizations induced by the coupling to the electrodes and that due to external phonon bath. The former one gives rise to a renormalized level detuning, which enhances both Fano factor and skewness uniquely to a super-Poissonian value, whereas does not affect the average current at all. For the latter, i.e., the phonon bath coupling, it introduces an intriguing interdot coupling renormalization, causing strong electron localization in QD2. Furthermore, the asymmetry in phonon absorption and emission leads to an asymmetric shot noise spectrum as well as a change of line shape in the skewness.

The remainder of this Letter is organized as follows. Based on the number-resolved reduced density matrix, the theory of FCS is outlined in SectionII. We then introduce in Section $\amalg$ the model Hamiltonian for the transport double dots coupled to a heat bath. The physical origin of the internal energy renormalizations is discussed in Section IV, along with its influence on the FCS analyzed. Finally, all the results are summarized in Section $\mathrm{V}$.

\section{FCS FORMALISM}

Full information about transport properties of a given system is contained in the probability distribution $P\left(N, t_{\mathrm{c}}\right)$ that $N$ electrons have transferred through the 
system during the counting time $t_{\mathrm{c}}$. The FCS yields all the cumulants $\left\langle I^{k}\right\rangle$ of the probability distribution, which are directly related to important properties of the junction. For instance, the first cumulant $\langle I\rangle$ gives the average current. The shot noise is related to the second cumulant $\left\langle I^{2}\right\rangle$ and is commonly represented by the Fano factor $F \equiv\left\langle I^{2}\right\rangle /\langle I\rangle$, with $F<1$ indicating a sub-Poisson noise, $F=1$ a Poisson fluctuation, and $F>1$ a superPoisson process. The third cumulant $\left\langle I^{3}\right\rangle$ measures the skewness of the distribution.

The cumulants can be found by performing derivatives of the cumulant generating function $(\mathrm{CGF}) \mathcal{F}(\chi)$ with respect to the counting field $\chi$,

$$
\left\langle I^{k}\right\rangle=-\left.\frac{1}{t_{\mathrm{c}}}\left(-\mathrm{i} \partial_{\chi}\right)^{k} \mathcal{F}\left(\chi, t_{\mathrm{c}}\right)\right|_{\chi=0},
$$

where the CGF is defined as

$$
e^{-\mathcal{F}\left(\chi, t_{\mathrm{c}}\right)} \equiv \sum_{N} P\left(N, t_{\mathrm{c}}\right) e^{\mathrm{i} N \chi}
$$

The involving probability distribution is closely related to the dynamics of the reduced system $\rho(t)$, which is obtained from the density matrix of the entire system by integrating out the reservoir degrees of freedom. To characterize the FCS, we further unravel $\rho(t)$ into components $\rho^{(N)}(t)$, in which ' $N$ ' denotes the number of electrons transferred through the system. The probability distribution is obtained via $P(N, t)=\operatorname{Tr} \rho^{(N)}(t)$, where $\operatorname{Tr}(\cdots)$ denotes the trace over the reduced system degrees of freedom.

To proceed, instead of using $\rho^{(N)}(t)$ directly, we shall employ its $\chi$-space counterpart, i.e., $\varrho(\chi, t) \equiv$ $\sum_{N} \rho^{(N)}(t) e^{\mathrm{i} N \chi}$. The corresponding quantum master equation reads formally [36-39]

$$
\frac{\partial}{\partial t} \varrho(\chi, t)=-\left(\mathrm{i} \mathcal{L}+\mathcal{R}_{\chi}\right) \varrho(\chi, t) \equiv \mathcal{L}_{\chi} \varrho(\chi, t),
$$

where $\mathcal{L}(\cdots) \equiv\left[H_{\text {sys }},(\cdots)\right]$ is the reduced system Liouvillian, $\mathcal{R}_{\chi}$ is the dissipation superoperator, and $\mathcal{L}_{\chi} \equiv$ $-\left(\mathrm{i} \mathcal{L}+\mathcal{R}_{\chi}\right)$, which will be specified later in the localized state representation [see Eq. (8) ].

With the knowledge of $\varrho(\chi, t)$, the CGF is determined straightforwardly as $\mathcal{F}\left(\chi, t_{\mathrm{c}}\right)=-\ln \left\{\operatorname{Tr} \varrho\left(\chi, t_{\mathrm{c}}\right)\right\}$. In the zero-frequency limit, i.e. the counting time $t_{\mathrm{c}}$ is much longer than the time of tunneling through the system, the CGF is simplified to [40 42 ]

$$
\mathcal{F}\left(\chi, t_{\mathrm{c}}\right)=-\lambda_{\min }(\chi) t_{\mathrm{c}},
$$

where $\lambda_{\min }(\chi)$ is the minimal eigenvalue of $\mathcal{L}_{\chi}$ that satisfies $\lambda_{\min }(\chi \rightarrow 0) \rightarrow 0$.

\section{MODEL DESCRIPTION}

Specifically, let us consider a double quantum dot system, as schematically shown in Fig.1, where the QD1

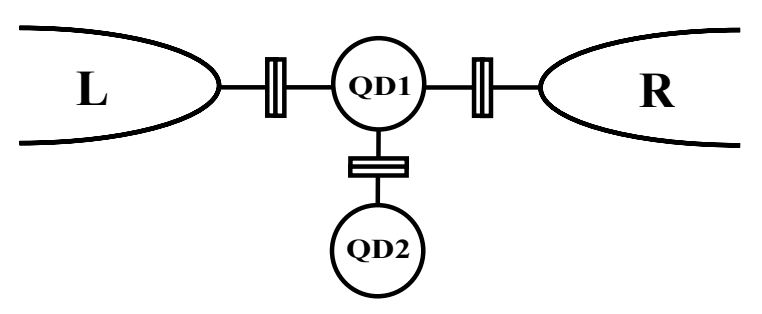

FIG. 1: Schematic setup for transport through a double quantum dot system, where QD1 is tunnel-coupled to the left and right electrodes, while QD2 is side-connected to the QD1. The system is further coupled to an inevitable dissipative phonon environment (not shown explicitly).

is connected to left and right electrodes, while the QD2 is side-connected to the QD1. The double dots are furthermore embedded in a dissipative phonon bath. The total Hamiltonian consists of the double dot system, the environment, and the coupling between them,

$$
H=H_{\mathrm{sys}}+H_{\mathrm{env}}+H_{\mathrm{sys}-\mathrm{env}} .
$$

Here the first part models the coupled dots

$$
\begin{aligned}
H_{\text {sys }}= & \sum_{\sigma}\left[\left(E_{0}+\frac{1}{2} \epsilon \hat{Q}_{z \sigma}\right)+\Omega \hat{Q}_{x \sigma}\right] \\
& +\sum_{\ell=1,2} U_{0} \hat{n}_{\ell \uparrow} \hat{n}_{\ell \downarrow}+U^{\prime} \hat{n}_{1} \hat{n}_{2},
\end{aligned}
$$

where $\hat{Q}_{z \sigma} \equiv d_{1 \sigma}^{\dagger} d_{1 \sigma}-d_{2 \sigma}^{\dagger} d_{2 \sigma}, \hat{Q}_{x \sigma} \equiv d_{1 \sigma}^{\dagger} d_{2 \sigma}+d_{2 \sigma}^{\dagger} d_{1 \sigma}$, $\hat{n}_{\ell}=\sum_{\sigma} \hat{n}_{\ell \sigma}$, and $\hat{n}_{\ell \sigma}=d_{\ell \sigma}^{\dagger} d_{\ell \sigma}$, with $d_{\ell \sigma}\left(d_{\ell \sigma}^{\dagger}\right)$ the electron annihilation (creation) operator in the QD1 $(\ell=1)$ or QD2 $(\ell=2)$ and spin $\sigma=\uparrow$ or $\downarrow$. Each dot consists of a single spin-degenerate energy level $E_{1 / 2}=E_{0} \pm \frac{1}{2} \epsilon$, measured relative to the equilibrium chemical potential of the electrodes. Electron tunneling between the two dots are characterized by the interdot tunneling strength $\Omega$. Double occupation on the same dot costs the intradot charging energy $U_{0}$. Simultaneous occupation of one electron in each dot is associated with the interdot charge energy $U^{\prime}$. Hereafter, the intradot charging energy $U_{0}$ is assumed to be much larger than the bias voltage, such that charge states with three or more electrons in the double dot are prohibited.

The environment is composed of the phonon bath, the left and right electrodes. The corresponding Hamiltonian reads $H_{\mathrm{env}}=h_{\mathrm{ph}}+h_{\mathrm{el}}$. Each of them is modeled as a collection of noninteracting particles. The phonon bath are modeled with $h_{\mathrm{ph}}=(1 / 2) \sum_{j} \hbar \omega_{j}\left(p_{j}^{2}+x_{j}^{2}\right)$. The electrodes assumes $h_{\mathrm{el}}=\sum_{\alpha=\mathrm{L}, \mathrm{R}} \sum_{k \sigma} \varepsilon_{\alpha k} c_{\alpha k \sigma}^{\dagger} c_{\alpha k \sigma}$, expressed in terms of electron creation $\left(c_{\alpha k \sigma}^{\dagger}\right)$ and annihilation $\left(c_{\alpha k \sigma}\right)$ operators in the left $(\alpha=\mathrm{L})$ or right $(\alpha=\mathrm{R})$ electrode. The electrodes are assumed to be in equilibrium, so that they are characterized by the Fermi distribution $f_{\mathrm{L} / \mathrm{R}}(\omega)$. An applied bias voltage $V$ is modeled by different chemical potentials in the left and right electrodes $\mu_{\mathrm{L} / \mathrm{R}}= \pm e V / 2$. 
The system-environment coupling can be written as $H_{\text {sys-env }}=H_{\text {sys-el }}+H_{\text {sys-ph }}$, with

$$
\begin{aligned}
H_{\mathrm{sys}-\mathrm{el}} & =\sum_{\alpha k \sigma}\left(t_{\alpha k} c_{\alpha k \sigma}^{\dagger} d_{1 \sigma}+\text { H.c. }\right), \\
H_{\mathrm{sys}-\mathrm{ph}} & =\sum_{\sigma} \hat{Q}_{z \sigma} F_{\mathrm{ph}} .
\end{aligned}
$$

Here $H_{\text {sys-el }}$ describes electron transfer between QD1 and the electrodes. $H_{\text {sys-ph }}$ models the coupling with phonon bath, in which $F_{\mathrm{ph}}=\sum_{j} \lambda_{j} x_{j}$. This term is responsible for the dot level energy fluctuations. The tunnel coupling between electrodes and QD1 is characterized by the coupling strength $\Gamma_{\alpha}=2 \pi \sum_{k}\left|t_{\alpha k}\right|^{2} \delta\left(\omega-\varepsilon_{\alpha k}\right)$. In what follows, we consider only spin conserving tunneling processes, and assume wide bands in the reservoirs, which yields energy independent couplings $\Gamma_{\alpha}$. The effect of phonon bath on the double dot system is characterized by the phonon interaction spectral density, $J_{\mathrm{ph}}(\omega)=\sum_{j}\left|\lambda_{j}\right|^{2} \delta\left(\omega-\omega_{j}\right)$. Hereafter, it is assumed to be Ohmic, i.e., $J_{\mathrm{ph}}(\omega)=\eta \omega e^{-\omega / \omega_{c}}$, where the dimensionless parameter $\eta$ reflects the strength of dissipation and $\omega_{\mathrm{c}}$ is the Ohmic high energy cutoff. Throughout this work, we set unit of $\hbar=e=1$ for the Planck constant and electron charge, unless stated otherwise.

\section{LEVEL RENORMALIZATION AND FCS ANALYSIS}

In the strong intradot Coulomb blockade regime, double occupation on the same dot is prohibited. The involving states are restricted to: $|0\rangle$-both dots empty, $|1 \sigma\rangle$-one electron in QD1, $|2 \sigma\rangle-$ one electron in QD2, and $\left|1 \sigma 2 \sigma^{\prime}\right\rangle$-one electron in each dot, respectively. The quantum master equation (3) in this localized state representation reads

$$
\begin{aligned}
& \varrho_{0}=-2\left(\Gamma_{\mathrm{L}}^{+}+\Gamma_{\mathrm{R}}^{+}\right) \varrho_{0}+\left(\Gamma_{\mathrm{L}}^{-}+\Gamma_{\mathrm{R}}^{-} e^{\mathrm{i} \chi}\right)\left(\varrho_{1 \uparrow}+\varrho_{1 \downarrow}\right), \\
& \dot{\varrho}_{1 \sigma}=\mathrm{i} \Omega\left(\varrho_{2 \sigma}^{1 \sigma}-\varrho_{1 \sigma}^{2 \sigma}\right)-\left(\Gamma_{\mathrm{L}}^{-}+\Gamma_{\mathrm{R}}^{-}\right) \varrho_{1 \sigma} \\
& \quad+\left(\Gamma_{\mathrm{L}}^{+}+\Gamma_{\mathrm{R}}^{+} e^{-\mathrm{i} \chi}\right) \varrho_{0}, \\
& \dot{\varrho}_{2 \sigma}=\mathrm{i} \Omega\left(\varrho_{1 \sigma}^{2 \sigma}-\varrho_{2 \sigma}^{1 \sigma}\right)-2\left(\tilde{\Gamma}_{\mathrm{L}}^{+}+\tilde{\Gamma}_{\mathrm{R}}^{+}\right) \varrho_{2 \sigma} \\
& \quad+\left(\tilde{\Gamma}_{\mathrm{L}}^{-}+\tilde{\Gamma}_{\mathrm{R}}^{-} e^{\mathrm{i} \chi}\right)\left(\varrho_{1 \sigma 2 \sigma}+\varrho_{1 \sigma} 2 \sigma\right), \\
& \dot{\varrho}_{1 \sigma 2 \sigma^{\prime}}=-\left(\tilde{\Gamma}_{\mathrm{L}}^{-}+\tilde{\Gamma}_{\mathrm{R}}^{-}\right) \varrho_{1 \sigma 2 \sigma^{\prime}}+\left(\tilde{\Gamma}_{\mathrm{L}}^{+}+\tilde{\Gamma}_{\mathrm{R}}^{+} e^{-\mathrm{i} \chi}\right) \varrho_{2 \sigma^{\prime}}, \\
& \dot{\varrho}_{2 \sigma}^{1 \sigma}=\mathrm{i}(\epsilon+\tilde{\epsilon}) \varrho_{2 \sigma}^{1 \sigma}+\mathrm{i}(\Omega+\tilde{\Omega})\left(\varrho_{1 \sigma}-\varrho_{2 \sigma}\right) \\
& \quad+\Gamma_{+} \varrho_{1 \sigma}-\Gamma_{-} \varrho_{2 \sigma}-\Gamma_{\mathrm{d}} \varrho_{2 \sigma}^{1 \sigma},
\end{aligned}
$$

with spin $\sigma \in\{\uparrow, \downarrow\}$ and $\bar{\sigma}=-\sigma$. Here $\varrho_{s}=\langle s|\varrho| s\rangle$ represents the diagonal element of the reduced density matrix. The off-diagonal elements $\varrho_{s^{\prime}}^{s}=\left\langle s|\varrho| s^{\prime}\right\rangle$ describes the so-called quantum "coherencies". The involving temperature-dependent tunneling rates are defined as $\Gamma_{\alpha}^{ \pm}=\Gamma_{\alpha} f_{\alpha}^{( \pm)}\left(E_{0}\right)$ and $\tilde{\Gamma}_{\alpha}^{ \pm}=\Gamma_{\alpha} f_{\alpha}^{( \pm)}\left(E_{0}+U^{\prime}\right)$, where $f_{\alpha}^{( \pm)}(\omega)=\left\{1+e^{ \pm \beta\left(\omega-\mu_{\alpha}\right)}\right\}^{-1}$ is related to the Fermi function of the electrode $\alpha=\mathrm{L}$ or $\mathrm{R}$, with $\beta=\left(k_{\mathrm{B}} T\right)^{-1}$. Here we are interested in the regime $\Delta<k_{\mathrm{B}} T\left(\Delta \equiv \sqrt{\epsilon^{2}+4 \Omega^{2}}\right.$ being the eigenenergy separation), where the external coupling strongly modifies the internal dynamics, and the off-diagonal elements of the reduced density matrix have vital roles to play [43, 44]. The level separation is thus smeared by the temperature and only the excitation energy levels $E_{0}$ and $E_{0}+U^{\prime}$ enter the Fermi functions.

The coupling to the phonon bath results in relaxation and dephasing in the system, with corresponding rates given by [45 48]

$$
\Gamma_{ \pm}=\frac{\eta \pi \Omega \epsilon}{\beta \Delta^{2}}-\frac{\pi}{2} \frac{\Omega}{\Delta} J_{\mathrm{ph}}(\Delta)\left[\frac{\epsilon}{\Delta} \operatorname{coth}\left(\frac{\beta \Delta}{2}\right) \pm 1\right],
$$

and

$$
\gamma_{\mathrm{ph}}=2 \pi \frac{\Omega^{2}}{\Delta^{2}} J_{\mathrm{ph}}(\Delta) \operatorname{coth}\left(\frac{\beta \Delta}{2}\right)+\frac{\eta \pi \epsilon^{2}}{\beta \Delta^{2}} .
$$

The total dephasing rate eventually reads

$$
\Gamma_{\mathrm{d}}=\frac{1}{2}\left(\Gamma_{\mathrm{L}}^{-}+\Gamma_{\mathrm{R}}^{-}\right)+\left(\tilde{\Gamma}_{\mathrm{L}}^{+}+\tilde{\Gamma}_{\mathrm{R}}^{+}\right)+\gamma_{\mathrm{ph}}
$$

By observing the equation of motion of the offdiagonal matrix element [Eq. [8el] ], it is found that the energy scales of the double dots are renormalized, i.e., $\epsilon \rightarrow \epsilon+\tilde{\epsilon}$ and $\Omega \rightarrow \Omega+\tilde{\Omega}$. The energy renormalizations $\tilde{\epsilon}$ and $\tilde{\Omega}$ are entailed respectively by coupling with the electrodes and phonon bath,

$$
\begin{aligned}
& \tilde{\epsilon}=\phi\left(E_{0}\right)-2 \phi\left(E_{0}+U^{\prime}\right)+\phi\left(E_{0}+U_{0}\right), \\
& \tilde{\Omega}=2 \pi \mathrm{i} \frac{\eta \Omega}{\beta \Delta}\{D(\Delta)-D(-\Delta)\},
\end{aligned}
$$

with

$$
\begin{aligned}
& \phi(\omega)=\sum_{\alpha=\mathrm{L}, \mathrm{R}} \frac{\Gamma_{\alpha}}{2 \pi} \operatorname{Re}\left[\Psi\left(\frac{1}{2}+\mathrm{i} \beta \frac{\omega-\mu_{\alpha}}{2 \pi}\right)\right], \\
& D(\omega)=e^{-\mathrm{i} \frac{\omega}{\omega_{c}}} B_{x}(y,-1)-e^{\mathrm{i} \frac{\omega}{\omega_{c}}} B_{x}\left(y^{*},-1\right) .
\end{aligned}
$$

Here, $\Psi$ and $B_{x}(y, z)$ are respectively digamma function and incomplete beta function [49], with $x=e^{-\frac{2 \pi}{\beta \omega_{c}}}$ and $y=1-\mathrm{i} \frac{\beta \omega}{2 \pi}$.

The detuning renormalization $\tilde{\epsilon}$ has been neglected in previous works [43, 44, 50-52], where the Fermi energies of the electrodes are assumed to be far away from the electronic states of the dots. The effect of interdot coupling renormalization, to our knowledge, has not yet been addressed in the context of electron quantum transport.

To clearly address the interdot coupling renormalization, we shall employ the quantum master equation (3), in which the dissipative term contains the contribution from the phonon bath

$$
\mathcal{R}_{\mathrm{ph}} \varrho(\chi, t)=\left[\hat{Q}_{z}, \tilde{Q}_{z} \varrho(\chi, t)\right]+\text { H.c. }
$$

with $\hat{Q}_{z}=\sum_{\sigma} \hat{Q}_{z \sigma}$ and

$$
\tilde{Q}_{z}=[\tilde{C}(-\mathcal{L})+\mathrm{i} \tilde{D}(-\mathcal{L})] \hat{Q}_{z} .
$$



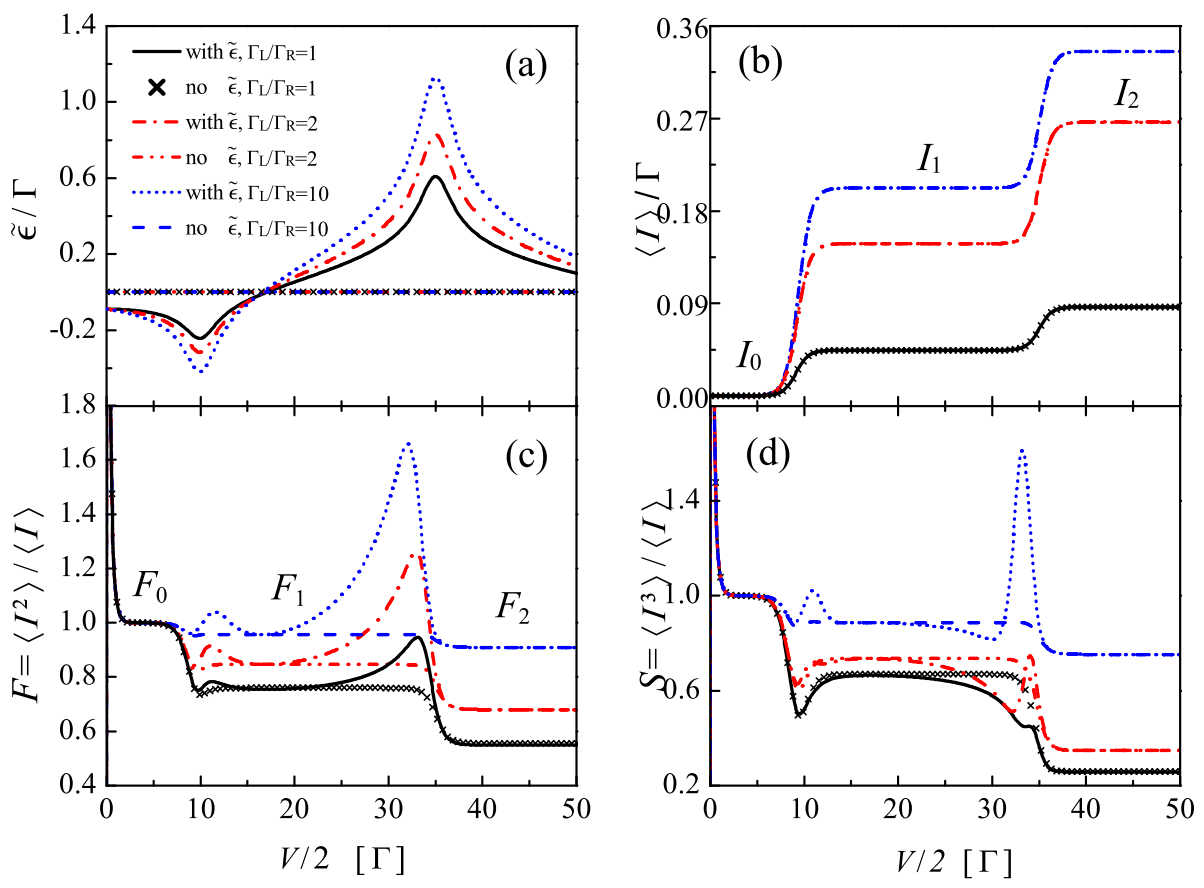

FIG. 2: (a) level detuning renormalization, (b) average current $\langle I\rangle$, (c) Fano factor $F=\left\langle I^{2}\right\rangle /\langle I\rangle$, and (d) normalized skewness $S=\left\langle I^{3}\right\rangle /\langle I\rangle$ versus the bias voltage for different $\Gamma_{\mathrm{L}} / \Gamma_{\mathrm{R}}$ ratios. Each time when the chemical potential of the electrode aligns with the energy needed for either single $\left(E_{0}\right)$ or double occupation $\left(E_{0}+U^{\prime}\right), \tilde{\epsilon}$ reaches its local extremum. Plot parameters are: $\epsilon=0, \Gamma \equiv \Gamma_{\mathrm{L}}+\Gamma_{\mathrm{R}}=2 \Omega, E_{0}=10 k_{\mathrm{B}} T, U^{\prime}=25 k_{\mathrm{B}} T$, and $U_{0}=60 k_{\mathrm{B}} T$.

Here, $\tilde{C}(-\mathcal{L})=\int_{-\infty}^{\infty} \mathrm{d} t C(t) e^{-\mathrm{i} \mathcal{L} t}$ is spectral function. The involving phonon bath correlation function is $C(t)=$ $\left\langle F_{\mathrm{ph}}(t) F_{\mathrm{ph}}(0)\right\rangle_{\mathrm{B}}$, with $\langle\cdots\rangle \equiv \operatorname{Tr}_{\mathrm{B}}\left[(\cdots) \rho_{\mathrm{B}}\right]$, and $\rho_{\mathrm{B}}$ the thermal equilibrium state of the phonon bath. The dispersion function $\tilde{D}(-\mathcal{L})$ involved can be evaluated via the Kramers-Kronig relation $\tilde{D}(-\mathcal{L})=-\frac{1}{\pi} \mathcal{P} \int_{-\infty}^{\infty} \mathrm{d} \omega \frac{C(\omega)}{\mathcal{L}-\omega}$, where $\mathcal{P}$ denotes the principal value. This term is responsible for the energy renormalization [12]. Let us focus on the last term in Eq. (14), which can be further expressed as

$\mathrm{i} \tilde{D}(-\mathcal{L}) \hat{Q}_{z}=4 \pi \mathrm{i} \frac{\eta \Omega^{2}}{\beta \Delta^{2}}[D(\Delta)+D(-\Delta)] \hat{Q}_{z}+\tilde{\Omega} \hat{Q}_{+}-\tilde{\Omega} \hat{Q}_{-}$,

with $\hat{Q}_{+}=|1\rangle\langle 2|$ and $\hat{Q}_{-}=|2\rangle\langle 1|$. Apparently, the first term accounts for the renormalization of level detuning. However, due to the odd function $D(\omega)$, this term eventually vanishes. The last two terms represent phonon absorption and emission. It is this phonon-induced effective coupling that gives rise to a unique interdot coupling modification which takes place only in Eq. (8e).

Hereafter, we will show that the both $\tilde{\epsilon}$ and $\tilde{\Omega}$ have important roles to play in the reduced dynamics, and consequently give rise to intriguing features in the high order cumulants of the current distribution. Consider first the situation without electron-phonon interaction $(\eta=0)$. The renormalization of interdot coupling $\tilde{\Omega}$ is thus zero. The numerical result for $\tilde{\epsilon}$ vs bias voltage is

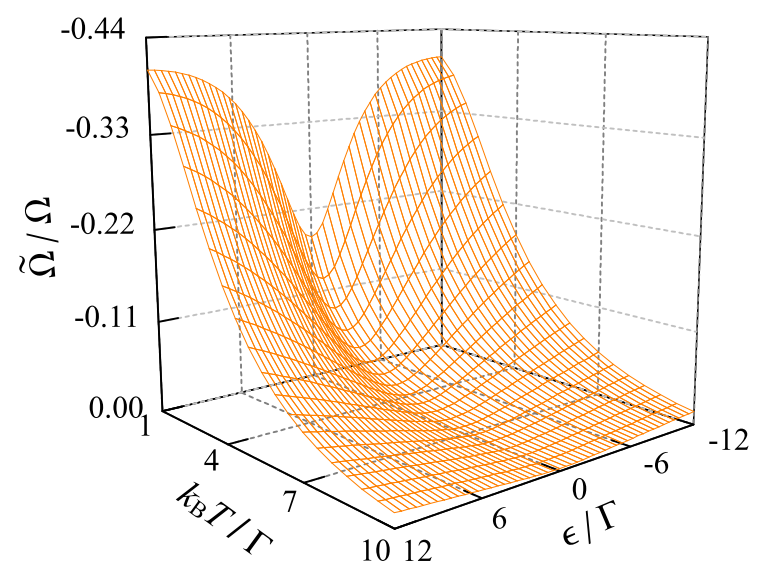

FIG. 3: $3 \mathrm{D}$ plot of the interdot coupling renormalization $\tilde{\Omega}$ vs bare level detuning $\epsilon$ and temperature $k_{\mathrm{B}} T$. The plotting parameters are $\Gamma_{\mathrm{L}}=\Gamma_{\mathrm{R}}=\Gamma / 2$, and $\eta=0.04, \omega_{\mathrm{c}}=15 \Gamma$ for the Ohmic phonon bath spectral density.

shown in Fig.2(a) for different $\Gamma_{L} / \Gamma_{R}$ ratios. Once the Fermi energy of the electrode is resonant with the energy needed for single $\left(E_{0}\right)$ or double occupation $\left(E_{0}+U^{\prime}\right), \tilde{\epsilon}$ reaches its local extremum. For a fixed total tunneling width $\Gamma=\Gamma_{\mathrm{L}}+\Gamma_{\mathrm{R}}, \tilde{\epsilon}$ increases whenever the tunnelcoupling asymmetry $\Gamma_{\mathrm{L}} / \Gamma_{\mathrm{R}}$ increases. 
Let us now investigate the influence of level detuning renormalization on FCS. The first three cumulants of the transport current are displayed in Fig.2(b), (c), and (d), respectively, as functions of the bias voltage. Here, the results with bare level detuning $(\tilde{\epsilon}=0)$ are also plotted for comparison.

The first cumulant, i.e., average current shows a typical step-like structure, as displayed in Fig.2(b). In the low bias voltage regime $\left(\mu_{\mathrm{L}}, \mu_{\mathrm{R}}<E_{0}\right)$, transport is exponentially suppressed, as the Fermi energies of both electrodes are well below the single level $E_{0}$. There are only very few thermally activated tunneling events, and the current $I_{0} \approx 0$. Transport through the system becomes energetically allowed when the Fermi level crosses the discrete level, i.e., $\mu_{\mathrm{L}}>E_{0}>\mu_{\mathrm{R}}$. The current rises to the second plateau. The bias voltage, however, is not sufficient to overcome the intradot and interdot charging energies, and the coupled dots can accommodate at most one electron. This is the so-called double-dot Coulomb blockade (DDCB) regime [38, 48]. The stationary current is obtained by utilizing Eq. (1)

$$
I_{1}=\frac{2 \Gamma_{\mathrm{L}} \Gamma_{\mathrm{R}}}{4 \Gamma_{\mathrm{L}}+\Gamma_{\mathrm{R}}},
$$

where the involving Fermi functions are approximated by either one or zero. The effective quadrupling of $\Gamma_{\mathrm{L}}$ is a combined effect of spin degeneracy and DDCB [37].

As the bias further increase and cross interdot charging energy, i.e., $\mu_{\mathrm{L}}>E_{0}+U^{\prime}, E_{0}>\mu_{\mathrm{R}}$, double occupancy (with one electron in each dot) is possible. It is also referred to as single-dot Coulomb blockade (SDCB) regime. The current eventually rises to the third plateau as shown in Fig.2(b), with its value given by

$$
I_{2}=\frac{2 \Gamma_{\mathrm{L}} \Gamma_{\mathrm{R}}}{2 \Gamma_{\mathrm{L}}+\Gamma_{\mathrm{R}}}
$$

where the effective doubling of $\Gamma_{L}$ arises from the spin degeneracy and SDCB [37].

Noticeably, the current is not sensitive at all to the level detuning renormalization $\tilde{\epsilon}$. The reason will be provided later. In order to further exploit the effect of the energy renormalization, we resort to higher order cumulants. The numerical result of the Fano factor against the bias voltage is displayed in Fig.2(c). At very small bias $V \ll k_{\mathrm{B}} T$, it is dominated by thermal noise, which is described by the well-known hyperbolic cotangent behavior, and eventually leads to a divergence of the Fano factor at $V=0$ [53, 54]. As the bias increases but still below $E_{0}$, there are only thermally activated uncorrelated tunneling events, and Fano factor is Poissonian, i.e., $F_{0}=1$. In the DDCB regime, electron transport through the bare level $E_{0}$ is energetically allowed. The Fano factor is given by

$$
F_{1}=1-\frac{8 \Gamma_{\mathrm{L}} \Gamma_{\mathrm{R}}}{\left(4 \Gamma_{\mathrm{L}}+\Gamma_{\mathrm{R}}\right)^{2}}+\frac{2 \Gamma_{\mathrm{L}}^{2}\left(\Gamma_{\mathrm{R}}^{2}+4 \tilde{\epsilon}^{2}\right)}{\left(4 \Gamma_{\mathrm{L}}+\Gamma_{\mathrm{R}}\right)^{2} \Omega^{2}},
$$

where the second term describes the suppression of the Fano factor below unity, whereas the third term gives a positive contribution. Unambiguously, the Fano factor is modulated by the detuning renormalization $\tilde{\epsilon}$. This shows the shot noise as a much more sensitive tool to the system parameters than the average current.

The level detuning renormalization gives rise to intriguing features in the noise spectrum. By neglecting $\tilde{\epsilon}$, the noise in the DDCB regime is constant against the bias. It can reach maximally the Poissonian value (for the chosen parameters here $\Gamma_{\mathrm{L}}+\Gamma_{\mathrm{R}}=2 \Omega$ ) in the limit of large tunnel-coupling asymmetry, i.e., $\Gamma_{\mathrm{L}} / \Gamma_{\mathrm{R}} \gg 1$. The detuning renormalization enhance the Fano factor in a unique way, i.e., the noise are increased at bias close to excitation energies $E_{0}$ and $E_{0}+U^{\prime}$. Remarkably, a pronounced super-Poissonian noise is observed provided tunnel couplings are sufficient asymmetric, as shown by the dotted curve in Fig.2(c).

To further understand the current and noise features, we perform a unitary transformation of the entire Hamiltonian, such that the system Hamiltonian is diagonalized to (here spin indices are suppressed for simplicity)

$$
\tilde{H}_{\text {sys }}=\frac{1}{2} \tilde{\Delta}\{|+\rangle\langle+|-|-\rangle\langle-|\},
$$

where $\tilde{\Delta} \equiv \sqrt{\tilde{\epsilon}^{2}+4 \Omega^{2}}$, with the detuning renormalization $\tilde{\epsilon}$ being appropriately taken into account. The coupled dot system can now be mapped onto a parallel twolevel system. Here, the bonding state $|+\rangle$ and the antibonding state $|-\rangle$ are defined respectively as

$$
\begin{aligned}
|+\rangle & =\sin \frac{\theta}{2}|2\rangle+\cos \frac{\theta}{2}|1\rangle, \\
|-\rangle & =\cos \frac{\theta}{2}|2\rangle-\sin \frac{\theta}{2}|1\rangle,
\end{aligned}
$$

where $\theta$ is introduced via $\sin \theta=2 \Omega / \tilde{\Delta}$ and $\cos \theta=\tilde{\epsilon} / \tilde{\Delta}$. As a result, the electron tunnel-coupling Hamiltonian (7a) is recast to

$$
\tilde{H}_{\text {sys-el }}=\sum_{\alpha k}\left\{t_{\alpha k}^{+} c_{\alpha k}^{\dagger}|0\rangle\left\langle+\left|+t_{\alpha k}^{-} c_{\alpha k}^{\dagger}\right| 0\right\rangle\langle-|\right\}+\text { H.c. }
$$

with $t_{\alpha k}^{+}=t_{\alpha k} \cos \frac{\theta}{2}$ and $t_{\alpha k}^{-}=t_{\alpha k} \sin \frac{\theta}{2}$. Apparently, the amplitudes for tunneling through the eigenstates $|+\rangle$ and $|-\rangle$ are effectively modulated by the detuning renormalization $\tilde{\epsilon}$.

The stationary current in general can be written as

$$
I=\int \frac{d \omega}{2 \pi} \mathcal{T}(\omega)\left\{f_{\mathrm{L}}(\omega)-f_{\mathrm{R}}(\omega)\right\},
$$

where $\mathcal{T}(\omega)$, the transmission probability, defines the probability for an incoming electron to be transmitted through the device. For a parallel two-level system in the DDCB regime, the transmission probability is related to

$$
\begin{aligned}
\mathcal{T} & \sim \frac{\left(\left|t_{\mathrm{L} k}^{+}\right|^{2}+\left|t_{\mathrm{L} k}^{-}\right|^{2}\right)\left|t_{\mathrm{R} k}^{+} t_{\mathrm{R} k}^{-}\right|^{2}}{\left|t_{\mathrm{R} k}^{+} t_{\mathrm{R} k}^{-}\right|^{2}+2\left|t_{\mathrm{R} k}^{+} t_{\mathrm{L} k}^{-}\right|^{2}+2\left|t_{\mathrm{R} k}^{-} t_{\mathrm{L} k}^{+}\right|^{2}} \\
& =\frac{2\left|t_{\mathrm{L} k}\right|^{2}\left|t_{\mathrm{R} k}\right|^{2}}{4\left|t_{\mathrm{L} k}\right|^{2}+\left|t_{\mathrm{R} k}\right|^{2}},
\end{aligned}
$$


where the $\theta$-dependence of the tunneling amplitudes cancel out. Eventually, it leads to the current expression of Eq. (16). Physically, suppression of electron tunneling through the anti-bonding state due to level renormalization is exactly compensated by that through the bonding state. The total transmission probability thus remains unchanged, and finally the current turns out to be detuning renormalization independent.

The Fano factor, however, is more sensitive to the internal dynamics, and reveals much detailed information about transport. Particularly, in the presence of large $\tilde{\epsilon}$, electron tunneling through the anti-bonding state is suppressed, while that through bonding state is enhanced. In the limit where the Coulomb interactions prevent a double occupancy of system, there is competition between the two transport channels. Consequently, the slow flowing of electrons through the bonding state modulates that through the fast state, giving rise to a bunching of tunneling events and eventually resulting in super-Poissonian noise, as displayed by the dotted curve in Fig.2(c).

Different from the Fano factor, the skewness can be either increased or reduced by $\tilde{\epsilon}$. In the case of symmetric tunnel-coupling $\left(\Gamma_{\mathrm{L}}=\Gamma_{\mathrm{R}}\right)$, the level renormalization leads to a suppression of skewness, see the solid curve in Fig.2(2(d). As the coupling asymmetry grows, $\tilde{\epsilon}$ can strongly enhance the skewness to a super-Poissonian value, and pronounced noise peaks are observed at bias close to $E_{0}$ and $E_{0}+U^{\prime}$, as shown by the dotted curve in Fig.2(d). The peak width of skewness, however, is reduced remarkably, compared with that of Fano factor.

We now investigate the effect of energy renormalization induced by external phonon bath. Let us ficus on the DDCB regime, i.e., at most one electron can reside on the double dot system, and investigate the effect of $\tilde{\Omega}$ based on FCS. One can always choose an appropriate bias voltage, at which the level detuning renormalization is zero, i.e., $\tilde{\epsilon}=0$, such that its influence is eliminated. In Fig. 3, $\tilde{\Omega}$ is plotted as a function of temperature $k_{\mathrm{B}} T$ and bare level detuning $\epsilon$. It is observed that the interdot coupling renormalization is fully negative; it thus reduces the total interdot coupling. It reaches a local minimum at $\epsilon=0$, and grows with increasing $\epsilon$. $\tilde{\Omega}$ is particularly notable at low temperature, but falls off rapidly with rising temperature.

The calculated Fano factor and normalized skewness are shown in Fig. प(a) and (b), respectively, as a function of the bare level detuning at different temperatures. For comparison, the results without $\tilde{\Omega}$ are also plotted. In the absence of the phonon bath $(\eta=0)$, both Fano factor and skewness are symmetric around $\epsilon=0$ (see the solid curves in Fig.(4). With nonzero coupling to the heat bath, phonon absorption and emission take place, which dominate for $\epsilon<0$ and $\epsilon>0$, respectively. At low temperatures, the asymmetry in emission and absorption leads to the observed asymmetric spectra in Fig.4. The coupling to the phonon bath furthermore causes dephasing between the two dot-levels, which generally suppresses the noise [46, 55]. The reduction of Fano factor
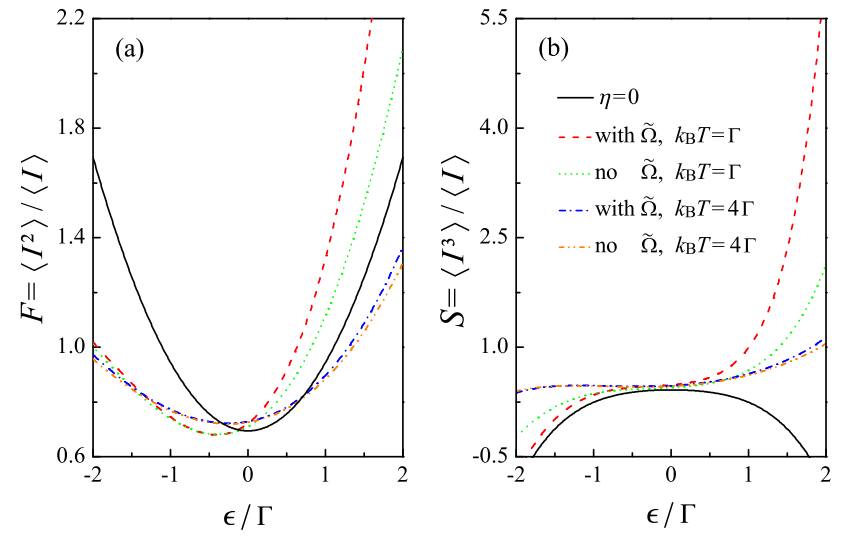

FIG. 4: (a) Fano factor and (b) normalized skewness in the DDCB regime vs bare level detuning for different temperatures. The other plotting parameters are the same as those in Fig. 3.

is particularly notable for $\epsilon<0$, as shown in Fig. प(a). On the other hand, electron-phonon scattering results in electron localization in QD2 [48]. In particular, for $\epsilon>0$ and at low temperature (e.g. $k_{\mathrm{B}} T=\Gamma$ ) this mechanism dominates. Electrons thus tend to be transferred in bunches, which enhances the noise in comparison with the case of $\eta=0$, as displayed by the dotted curves in Fig.4(a).

Finite $\tilde{\Omega}$ (due to its negative contribution) suppresses electron tunneling between the two dots, and effectively increases electron localization in QD2. Thereby the noise is enhanced, particularly at low temperature, where $\tilde{\Omega}$ is most prominent [see the dashed curves in Fig.4(a)]. Noise enhancement due to weak interdot coupling were also reported in Ref. [56], where counting statistics for electron transport through a double-dot AharonovBohm interferometer was investigated. However, there the divergence is closely related to a separation of the Hilbert space of the double dots into disconnected subspaces that contain the spin singlet and triplet states for double occupancy.

The effect of phonon bath on the third order cumulant is displayed in Fig.4(b), where the normalized skewness is plotted against the bare detuning $\epsilon$. Whenever the double dots are coupled to the phonon bath, the skewness is enhanced and becomes asymmetric. Noticeably, above the resonance $(\epsilon>0)$ a change of line shape is observed, i.e., the skewness increases with growing $\epsilon$, rather than decreases as in the case of $\eta=0$. This intriguing feature shows unambiguously the third order cumulant as a much sensitive tool to the phonon bath coupling. The presence of finite $\tilde{\Omega}$ furthermore increases the skewness, especially at low temperature [see the dashed curve in Fig. प(b)].

With the knowledge of energy renormalizations $\tilde{\epsilon}$ and $\tilde{\Omega}$, we are now in a position to consider their combined effect. The Fano factor is plotted as a function of bias voltage and temperature in Fig.5. In the lower half of 


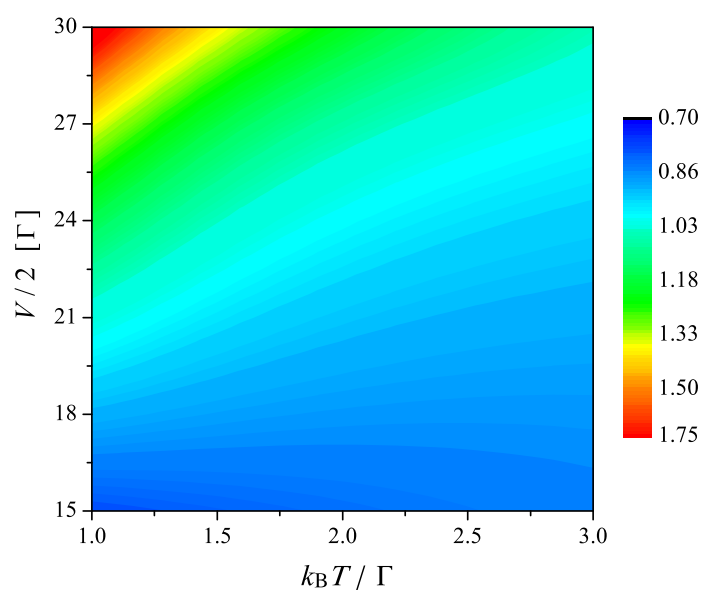

FIG. 5: Contour plot of the Fano factor versus the bias and bare level detuning in the DDCB regime. The plotting parameters are, $\Gamma_{\mathrm{L}}=2 \Gamma_{\mathrm{R}}$ and $\epsilon=0$. The parameters for the phonon bath are the same as those in Fig. 3

the bias regime, say $V / 2<20 \Gamma$, the level detuning renormalization $\tilde{\epsilon}$ is not strong [see Fig.2(a)]. The Fano factor thus exhibits sub-Poissonian statistics. Increase of $\tilde{\epsilon}$ due to rising bias voltage results in noise enhancement, and consequently super-Poissonian noises are observed in the upper half bias regime. Noise is further increased at low temperatures, where the interdot coupling renormalization $\tilde{\Omega}$ effectively suppresses tunneling between the two dots and electrons tend to be transferred in bunches more readily.

\section{CONCLUSION}

In summary, we have investigated the full counting statistics of electron transport through a side-connected double quantum dot system, and paid particular attention to the intriguing features arising from the renormalized internal dynamics. It is found that, in comparison with the average current which is not sensitive at all to the detuning renormalization, the Fano factor and normalized skewness are both enhanced to a super-Poissonian value in a unique way. Phonon emission and absorption take place when the double dots are further coupled to an external heat bath, and give rise to an intriguing interdot coupling renormalization. Due to negative contribution, it effectively localizes electron in QD2, which eventually leads to strong enhancement of the noise spectra. The asymmetry of phonon emission and absorption processes result in an asymmetric Fano factor, and a change of line shape in the skewness. Our investigations demonstrate unambiguously the importance of the internal energy renormalizations, which thereby should be properly accounted for in transport through various nanoscale devices.

\section{Acknowledgments}

Support from the National Natural Science Foundation of China (10904128), Zhejiang Provincial Natural Science Foundation (Y6110467), and UGC (AOE/P-04/082 ) and RGC (604709) of Hong Kong SAR Government is gratefully acknowledged.
[1] S. Datta, Electronic Transport in Mesoscopic Systems, Oxford University Press, New York, 1995.

[2] W. Lu, Z. Ji, L. Pfeiffer, K. W. West, A. J. Rimberg, Real-time detection of electron tunnelling in a quantum dot, Nature 423 (2003) 422.

[3] G. Fève, A. Mahé, J.-M. Berroir, T. Kontos, B. Plaçais, D. C. Glattli, A. Cavanna, B. Etienne, Y. Jin, An on-demand coherent single-electron source, Science 316 (2007) 1169.

[4] K. Ono, D. G. Austing, Y. Tokura, S. Tarucha, Current rectification by pauli exclusion in a weakly coupled double quantum dot system, Science 297 (2002) 1313-1317.

[5] T. Hayashi, H. D. C. T. Fujisawa, Y. Hirayam, Coherent manipulation of electronic states in a double quantum dot, Phys. Rev. Lett. 91 (2003) 226804.

[6] J. R. Petta, A. C.Johnson, J. M.Taylor, E. A. Laird, A. Yacoby, M. D. Lukin, C. M. Marcus, M. P. Hanson, A. C. Gossard, Coherent manipulation of coupled electron spins in semiconductor quantum dots, Science 309 (2005) 2180.

[7] F. H. L. Koppens, C. Buizert, K. J. Tielrooij, I. T. Vink, K. C. Nowack, T. Meunier, L. P. Kouwenhoven, L. M. K. Vandersypen, Driven coherent oscillations of a single electron spin in a quantum dot, Nature 442 (2006) 766-771.
[8] T. Fujisawa, T. Hayashi, R. Tomita, Y. Hirayama, "s", Science 312 (2006) 1634.

[9] S. Gustavsson, R. Leturcq, M. Studer, T. Ihn, K. Ensslin, D. C. Driscoll, A. C. Gossard, Time-resolved detection of single-electron interference, Nano Lett. 8 (2008) 25472550 .

[10] A. J. Leggett, S. Chakravarty, A. T. Dorsey, M. P. A. Fisher, A. Garg, W. Zwerger, Dynamics of the dissipative two-state system, Rev. Mod. Phys. 59 (1987) 1-85, 67, 725-726(Erratum) (1995).

[11] U. Weiss, Quantum Dissipative Systems, World Scientific, Singapore, 2008, 3rd ed. Series in Modern Condensed Matter Physics, Vol. 13.

[12] Y. J. Yan, R. X. Xu, Quantum mechanics of dissipative systems, Annu. Rev. Phys. Chem. 56 (2005) 187-219.

[13] J. König, J. Martinek, Interaction-driven spin precession in quantum-dot spin valves, Phys. Rev. Lett. 90 (2003) 166602.

[14] M. Braun, J. König, J. Martinek, Frequency-dependent current noise through quantum-dot spin valves, Phys. Rev. B 74 (2006) 075328.

[15] B. Wunsch, M. Braun, J. König, D. Pfannkuche, Probing level renormalization by sequential transport through double quantum dots, Phys. Rev. B 72 (2005) 205319. 
[16] B. Sothmann, J. König, Transport through quantum-dot spin valves containing magnetic impurities, Phys. Rev. B 82 (2010) 245319.

[17] F. Marquardt, C. Bruder, Dephasing in sequential tunneling through a double-dot interferometer, Phys. Rev. B 68 (2003) 195305.

[18] C. Bruder, R. Fazio, H. Schoeller, Aharonov-bohm oscillations and resonant tunneling in strongly correlated quantum dots, Phys. Rev. Lett. 76 (1996) 114-117.

[19] J. Fransson, O. Eriksson, Current-voltage asymmetries and negative differential conductance due to strong electron correlations in double quantum dots, Phys. Rev. B 70 (2004) 085301.

[20] J. Fransson, O. Eriksson, Asymmetric negative differential conductance in double quantum dots, J. Phys.: Condens. Matter 16 (2004) L85-L91.

[21] J. Y. Luo, H. J. Jiao, F. Li, X.-Q. Li, Y. J. Yan, Reduced dynamics with renormalization in solid-state charge qubit measurement, J. Phys.: Cond. Matt. 21 (2009) 385801.

[22] J. Y. Luo, H. J. Jiao, J. Z. Wang, Y. Shen, X.-L. He, Renormalized dynamics in charge qubit measurements by a single electron transistor, Phys. Lett. A 374 (2010) 4904-4908.

[23] L. S. Levitov, H. W. Lee, G. B. Lesovik, Electron counting statistics and coherent states of electron current, J. Math. Phys. 37 (1996) 4845.

[24] D. A. Bagrets, Y. V. Nazarov, Full counting statistics of charge transfer in coulomb blockade systems, Phys. Rev. B 67 (2003) 085316.

[25] J. Bylander, T. Duty, P. Delsing, Current measurement by real-time counting of single electrons, Nature 434 (2005) 361-364.

[26] R. Schleser, E. Ruh, T. Ihn, K. Ensslin, D. C. Driscoll, A. C. Gossard, Time-resolved detection of individual electrons in a quantum dot, Appl. Phys. Lett. 85 (2004) 2005-2007.

[27] L. M. K. Vandersypen, J. M. Elzerman, R. N. Schouten, L. H. Willems van Beveren, R. Hanson, L. P. Kouwenhoven, Real-time detection of single-electron tunneling using a quantum point contact, Appl. Phys. Lett. 85 (2004) 4394-4396.

[28] S. Gustavsson, R. Leturcq, B. Simovic, R. Schleser, T. Ihn, P. Studerus, K. Ensslin, D. C. Driscoll, A. C. Gossard, Counting statistics of single electron transport in a quantum dot, Phys. Rev. Lett. 96 (2006) 076605.

[29] S. Gustavsson, R. Leturcq, M. Studer, I. Shorubalko, T. Ihn, K. Ensslin, D. Driscoll, A. Gossard, Electron counting in quantum dots, Surf. Sci. Rep. (2009) 64 (2009) 191-232.

[30] A. Gruneis, M. J. Esplandiu, D. Garcia-Sanchez, A. Bachtold, Detecting individual electrons using a carbon nanotube field-effect transistor, Nano Lett. 7 (2007) 3766-3769.

[31] A. Thielmann, M. H. Hettler, J. König, G. Schön, SuperPoissonian noise, negative differential conductance, and relaxation effects in transport through molecules, quantum dots, and nanotubes, Phys. Rev. B 71 (2005) 045341.

[32] S. Welack, J. B. Maddox, M. Esposito, U. Harbola, S. Mukamel, Single-electron counting spectroscopy: Simulation study of porphyrin in a molecular junction, Nano Lett. 8 (2008) 1137.

[33] W. G. van der Wiel, S. D. Franceschi, J. M. Elzerman, T. Fujisawa, S. Tarucha, L. P. Kouwenhoven, Elec- tron transport through double quantum dots, Rev. Mod. Phys. 75 (2003) 1.

[34] A. Nauen, I. Hapke-Wurst, F. Hohls, U. Zeitler, R. J. Haug, K. Pierz, Shot noise in self-assembled inas quantum dots, Phys. Rev. B 66 (2002) 161303.

[35] S. Sasaki, H. Tamura, T. Akazaki, T. Fujisawa, Fanokondo interplay in a side-coupled double quantum dot, LANL e-print arXiv:0912.1926.

[36] X.-Q. Li, J. Y. Luo, Y. G. Yang, P. Cui, Y. J. Yan, Quantum master-equation approach to quantum transport through mesoscopic systems, Phys. Rev. B 71 (2005) 205304.

[37] J. Y. Luo, X.-Q. Li, Y. J. Yan, Calculation of the current noise spectrum in mesoscopic transport: A quantum master equation approach, Phys. Rev. B 76 (2007) 085325.

[38] J. Y. Luo, X.-Q. Li, Y. J. Yan, Spin-dependent current noises in transport through coupled quantum dots, J. Phys.: Cond. Matt. 20 (2008) 345215.

[39] S.-K. Wang, H. Jiao, F. Li, X.-Q. Li, Y. J. Yan, Full counting statistics of transport through two-channel coulomb blockade systems, Phys. Rev. B 76 (2007) 125416.

[40] C. W. Groth, B. Michaelis, C. W. J. Beenakker, Counting statistics of coherent population trapping in quantum dots, Phys. Rev. B 74 (2006) 125315.

[41] C. Flindt, T. Novotny, A.-P. Jauho, Full counting statistics of nano-electromechanical systems, Europhys. Lett. 69 (2005) 475.

[42] G. Kießlich, P. Samuelsson, A. Wacker, E. Schöll, Counting statistics and decoherence in coupled quantum dots, Phys. Rev. B 73 (2006) 033312.

[43] S. A. Gurvitz, Y. S. Prager, Microscopic derivation of rate equations for quantum transport, Phys. Rev. B 53 (1996) 15932-15943.

[44] T. H. Stoof, Y. V. Nazarov, Time-dependent resonant tunneling via two discrete states, Phys. Rev. B 53 (1996) 1050-1053.

[45] T. Brandes, Coherent and collective quantum optical effects in mesoscopic systems, Phys. Rep. 408 (2005) 315474.

[46] G. Kießich, E. Schöll, T. Brandes, F. Hohls, R. J. Haug, Noise enhancement due to quantum coherence in coupled quantum dots, Phys. Rev. Lett. 99 (2007) 206602.

[47] R. Aguado, T. Brandes, Shot noise spectrum of open dissipative quantum two-level systems, Phys. Rev. Lett. 92 (2004) 206601.

[48] J. Y. Luo, S.-K. Wang, X.-L. He, X.-Q. Li, Y. J. Yan, Real-time counting of single electron tunneling through a t-shaped double quantum dot system, J. Appl. Phys. 108 (2010) 083720.

[49] I. S. Gradshteyn, I. M. Ryzhik, Table of Integrals, Series, and Products, Academic Press, New York, 1980, 4th ed.

[50] S. A. Gurvitz, Measurements with a noninvasive detector and dephasing mechanism, Phys. Rev. B 56 (1997) $15215-23$.

[51] S. A. Gurvitz, Rate equations for quantum transport in multidot systems, Phys. Rev. B 57 (1998) 6602-11.

[52] B. L. Hazelzet, M. R. Wegewijs, T. H. Stoof, Y. V. Nazarov, Coherent and incoherent pumping of electrons in double quantum dots, Phys. Rev. B 63 (2001) 165313.

[53] Y. M. Blanter, M. Büttiker, Phys. Rep. 336 (2000) 1.

[54] E. V. Sukhorukov, G. Burkard, D. Loss, Noise of a quantum dot system in the cotunneling regime, Phys. Rev. B 
$63(2001) 125315$.

[55] A. Braggio, C. Flindt, T. Novotný, The influence of charge detection on counting statistics, J. Stat. Mech. 1 (2009) 1048.
[56] D. Urban, J. König, Tunable dynamical channel blockade in double-dot Aharonov-Bohm interferometers, Phys. Rev. B 79 (2009) 165319. 